\title{
Equipment Support Training System Design
}

\author{
Wang Hui, Zhang Qin \\ Department of Command and Administration \\ Academy of Armored Force Engineering \\ Beijing 100072, China \\ zgyzhxwh@126.com
}

\begin{abstract}
This work describes the development of a novel equipment support training system. Differing from the traditional teaching panel method, the proposed method does not require any complex computations for coordinate transformation and is a simpler scheme. The proposed equipment support training system includes small equipment support training robots. The system specification phase determines the basic functionalities of the system, which can be described as a series of multi - level goals. Interface analysis is used to understand the input and output interactions between the system and the environment. The equipment support training system architecture, control board design and program flowchart are described and implemented.
\end{abstract}

Keywords-support training system; system architecture; embedded system

\section{INTRODUCTION}

Although robot manufacturers supply teaching programs for planning, the trajectories use training panels or joysticks. With these methods, the user moves the robot manually through the space by operating the manual box (teaching panel) and a series of processes are required. The user must have sufficient training, otherwise, it is difficult to complete the teaching task. These teaching methods are not sufficiently intuitive; that is, intuitive teaching schemes are required for industrial application. Notably, many robot applications do not require highly precise motion control. For example, an intuitive robot training system is needed for such applications as robotic baccarat dealers. Here, we present a novel equipment support training system design. The proposed equipment support training system includes small equipment support training robots[1-2]. The equipment support training system architecture, control board design and program flowchart are described and implemented.

\section{METHODOLOGY FOR EMBEDDED REALIZATION}

The Prometheus methodology is a three-phase agentoriented design process for software systems, and the design tool based on this methodology is also available for practical software development. Accordingly, it has been applied in the software development of a surveillance-related robotic. This methodology offers a collection of guidelines and means, e.g. goal overview, scenarios analysis and roles assignment, to help the designer to comprehend system functions and to determine the elements that form the MAS. It also explicitly considers agent perceptions and actions as modeling elements, and it can be used to explain why and how the different elements are obtained from agent - based applications. The Prometheus methodology comprises three phases, and we adapt its process for development by using the embedded system resources at the hardware and software level, as shown in Fig.1.

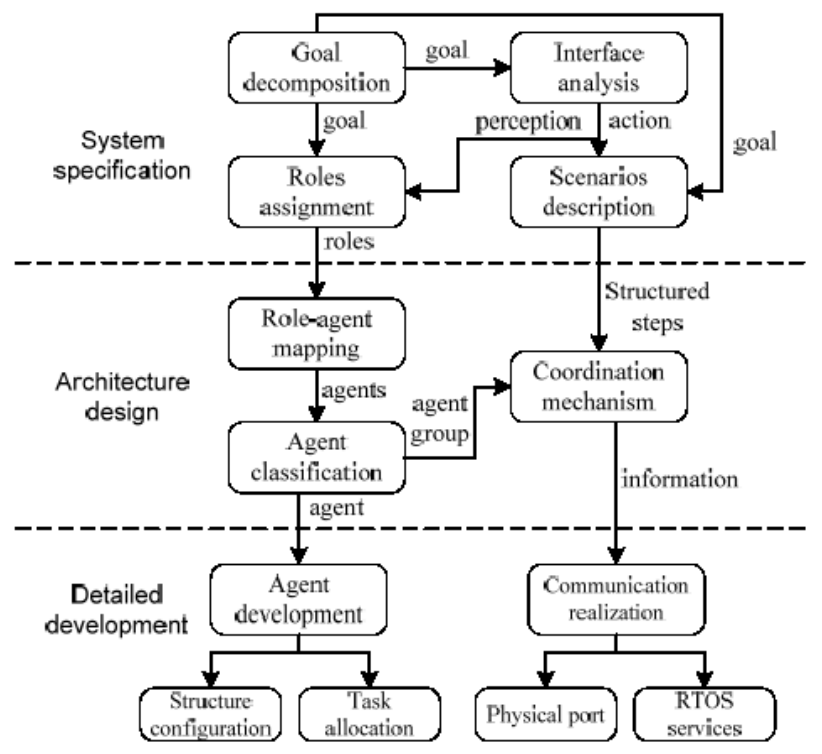

Figure 1. The methodology for embedded realization.

The system specification phase determines the basic functionalities of the system, which can be described as a series of multi - level goals. Interface analysis is used to understand the input and output interactions between the system and the environment, which can also be considered as the perception and action for a robotic system. The system operation status is expressed as a scenario that contains a sequence of structured job steps. Each autonomous element in a scenario is defined as a role, which can be played by an agent or several agents together.

The architecture design phase investigates the agent types that exist in the system and how they interact. The agents are identified by role-agent mapping, which analyses 
the correlation of goals, perceptions and actions of multiple roles. Agent types and numbers are designed to play the different roles. Their means of interaction are described in the MAS coordination mechanism [3].

\section{SYSTEM ARCHITECTURE}

\section{A. Electrical control system design}

The design of the electrical control system for the proposed teaching system consists mainly of a DC servo control card, a control motherboard, a teaching program design and a control program for each servo control card.

\section{1) DC servo control card design}

To preserve extension flexibility, each joint is driven by one servo control card. Each servo control card receives a pulse-width command to complete the position loop control, as does a radio control (RC) servo motor. The pulse width is proportional to the rotation angle and the ratio is adjustable depending on each joint's rotation range. Also, a tuned proportional-integral-derivative (PID) controller is implemented in the position loop to guarantee that the system has acceptable steady state error. Fig.2 shows a functional block diagram of the joint control card. The necessary peripheral interfaces, the control kernel, power MOSFET, current sensor and encoder counting circuit are integrated into the servo control card.

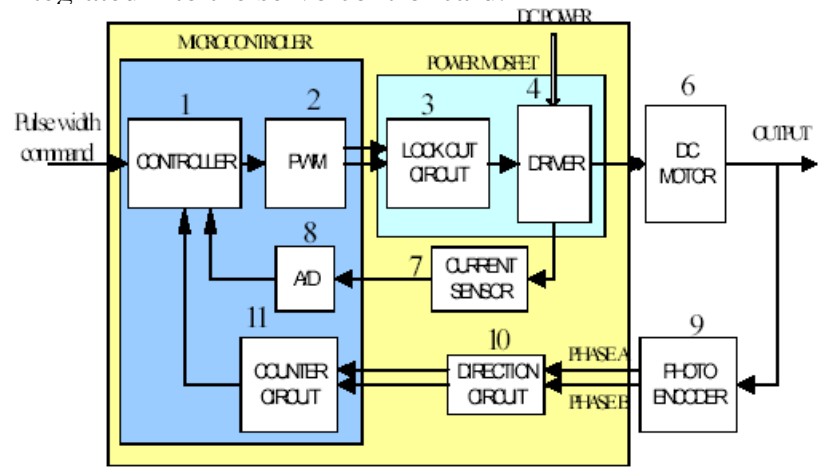

Figure 2. Schematic diagram of the dc servo control card.

\section{2) Mother control board design}

When in teaching mode, the control motherboard receives voltage signals from the teaching robot and then sends the related trajectory command to the DC servo control card. The real robot and teaching robot execute nearly the same motion. The operator moves the teaching robot and observes the real robot's motion to decide whether to continue dragging the teaching robot. All trajectory commands are recorded in memory and can be recalled to repeat a teaching action. Fig3. shows the functional block diagram of the control motherboard.

The mode indicator displays the operational mode, which includes a follow mode, demo mode and teaching mode. This work focuses on teaching mode. A 7-segment displayer shows the run time, indicating execution time for a teaching cycle. The pulse-width command block sends the PWM signals to the DC servo control card in sequence. The kernel of the control motherboard is dsPIC30F6014A. Other functions occur, including writing teaching signals to the extension memory 25AA1024, reading the potentiometer signals and transforming them into positional commands, detecting the push buttons to switch operational modes, receiving feedback signals coming from the DC servo control card in response to related actions, such as home position operation and deciding whether the position loop control is finished. The major functional blocks are described as follows:

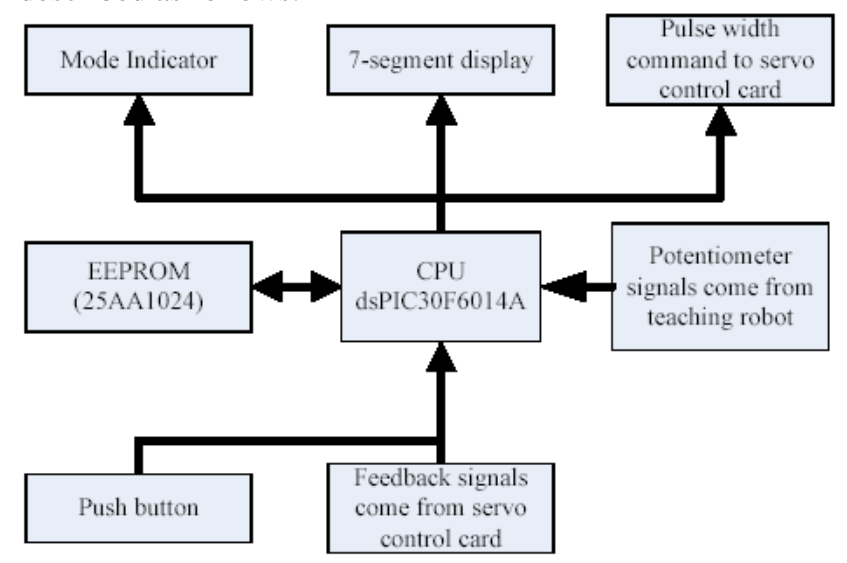

Figure 3. Schematic diagram of the mother control board.

3) Pulse - width command to the DC servo control card A novel five-joint robot teaching system was developed. Each joint is controlled by a DC servo control card. As described, the servo card receives the pulse-width command. For more axes joint robot systems, generating synchronous PWM signals generates smoother motion, but has higher start current from the power supply. To reduce the normal rated power, the motherboard sends a sequential pulse-width command to the DC servo control cards. Fig. 4 shows the sequential pulse-width commands and shows the schematic diagram of the pulse-width command generation [4].

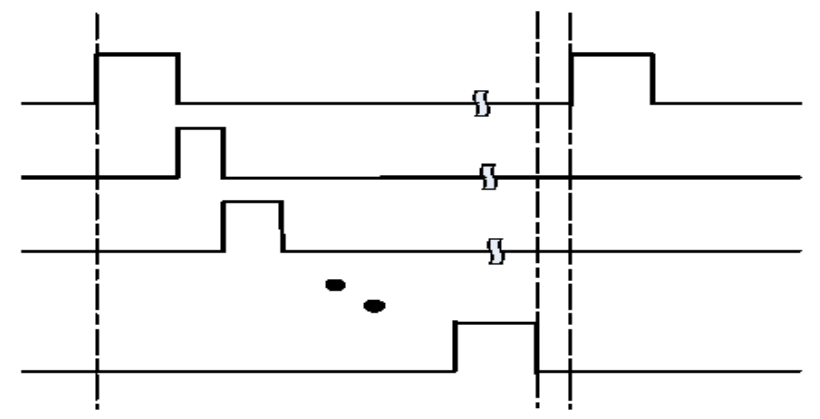

Figure 4. Sequential pulse width command.

\section{B. Programming of Training System}

\section{1) Mother control board program}

The control motherboard has three operational modes: follow mode, demo mode and teaching mode. In follow mode, the real robot mimics the motion of the teaching robot. Demo mode executes some special motions, such as dealing. Fig. 5 shows the flowchart of the teaching mode. Teaching 
signals coming from the potentiometers must be recorded in the non-volatile memory 25AA1024 in real time. To illustrate the read/write timing of the datasheet, Fig. 10 shows the read and write flowcharts. Notably, 25AA1024 has is only 1 Mbits of memory. Its recordable teaching time is related to the sampling time. Small sample times will generate high trajectory resolution. Each sample requires 10 bytes of memory for each 5 of joint movement. Each command occupies 2 bytes of memory for each joint. If $50 \mathrm{~ms}$ is taken as sampling time, 25AA1024 it can record roughly 625 seconds of path planning; longer path learning is easily completed by expanding the memory.

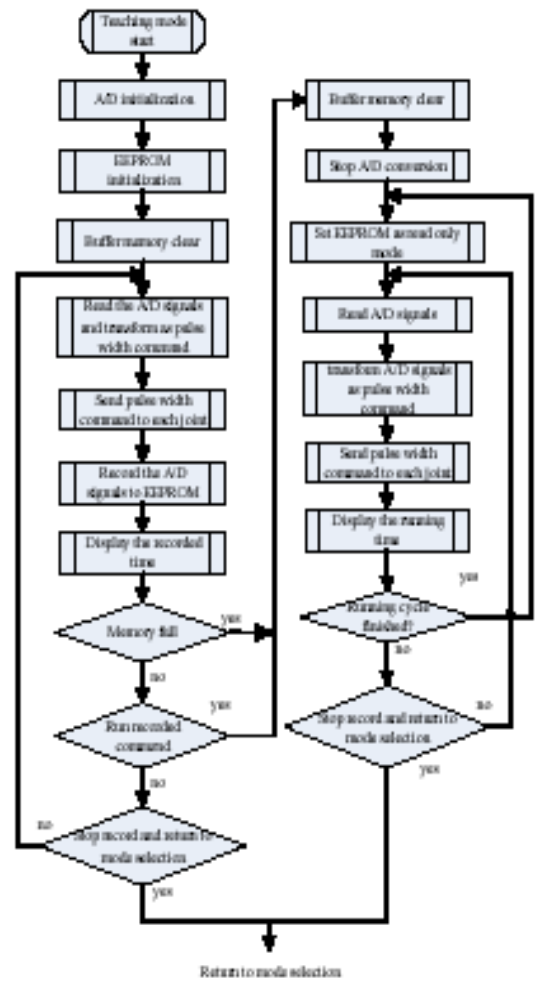

Figure 5. Flowchart of teaching mode program.

\section{2) Program design of the DC servo control card}

The DC servo control card receives a pulse-width command and then executes the closed - loop servo control. By using PIC18f8720 as the control kernel of the DC servo control card, PIC18f8720 needs peripheral initialization, including interrupt mode, capture mode, PWM mode and the necessary input/output $(\mathrm{I} / \mathrm{O})$ pins. After initialization, the kernel runs the main control loop, the shaded yellow area. The main control loop runs with 1 ms control cycles and the controller is PID type. Notably, the position loop control is similar to that of RC servo motor control. A special pulsewidth command corresponds to a motor position. Each servo control card receives a pulse-width command and calculates the pulse width via the capture module, and then completes the corresponding position loop control [5].

\section{TRAINING SYSTEM}

An educational system, particularly a remote class system, has been developed as a practical application of the android robot AFEI. In considering practical aspect of communication robot, to achieve smooth and natural communication between human and robot is one of the most important problems. However, intelligence technologies of robots are generally still lacked to interact with human and act in daily lives autonomously even though variety of autonomous robots have been developed and studied so far. Autonomous communication robots are currently simply able to interact with human in well-designed interaction scenarios and in well-defined environment as well.

Meanwhile, tele-operated robot which is manoeuvred by a hidden operator has the advantage in terms of practicality because it seems to conduct behaviors and interactions autonomously from the viewpoint of a human who interacts with the robot even though the robot is controlled by teleoperation. Particularly, if an android robot is used as an interface of tele-operated communication system, it will give people a strong feeling of presence and make people feel like they are interacting with real human. In addition, it is expected that elementary school students are very interested in interaction with an android robot and they actively participate in the class which is conducted by the android robot. The detailed configuration of the proposed educational system is described as follows.

\section{A. System Configuration}

The template is used to format your paper and style the text. All margins, column widths, line spaces, and text fonts are prescribed; please do not alter them. You may note peculiarities. For example, the head margin in this template measures proportionately more than is customary. This measurement and others are deliberate, using specifications that anticipate your paper as one part of the entire proceedings, and not as an independent document. Please do not revise any of the current designations.

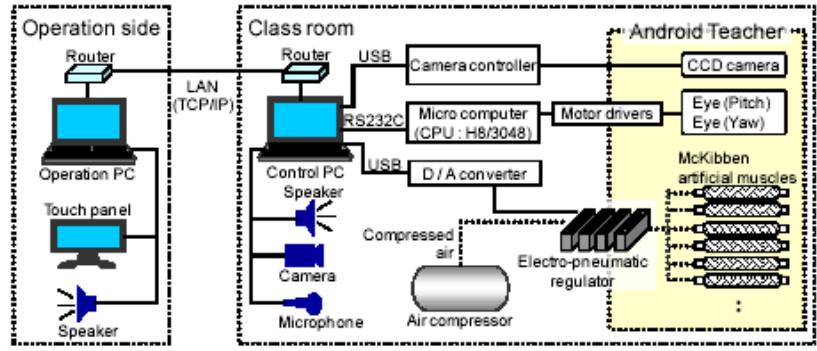

Figure 6. System configuration of remote class system with the android robot AFEI.

Fig. 6 shows the system configuration of the proposed training system, and the android robot AFEI is utilized as the role of a teacher. In the classroom, there are AFEI and some control equipment, and the control system of AFEI requires a compressor and an electro-pneumatic regulator to control contractions of McKibben artificial muscles. In addition, the electric-pneumatic regulator is controlled by the control 
computer (“Control PC”) to control AFEI's facial expressions and head movements, and the control computer also controls AFEI's eye-direction and utterances as well. A microphone and a video camera are used to obtain visual and sound information of the classroom.

In the operation room, there are two monitors. One of them is used for the control, and the other one is used for the observation. The operator is able to monitor students' behaviors through the observation monitor, and he can manoeuvre AFEI's utterances and actions by sending commands from the operation PC ("Operation PC") to the control PC ("Control PC") through the LAN. The control PC executes robot's utterances and actions based on received commands. Specifically, captured images from AFEI's CCD camera and the video camera are transmitted to the operation $\mathrm{PC}$, so the operator can observe the classroom from the two viewpoints. As a result, the operator can move AFEI's viewpoint by controlling its eye and head directions corresponding to these visual information, and AFEI is able to look around the classroom and look at a student. The operator is able to hear students from the speakers and respond to students as well [6].

\section{B. Interactive Behaviors}

In the developed system, there are two operation modes which include "lecture mode" and "interaction mode" . In "lecture mode" , AFEI gives some explanations about some contents of a class to students while looking around the classroom by the tele-operation, and some slides which are projected on the screen in front of the classroom to help students to understand simultaneously. AFEI's utterances are previously prepared along a scenario of a class. In "interaction mode", AFEI performs interactive behaviors such as looking around the room, paying attention to a student, and talking to a student. In order to talk to students, AFEI is able to respond with registered short sentences such as "Do your best!", “Be quiet!”,

"Don't look away" and so on. If students' questions are beyond AFEI's default database, AFEI replies with a word of kindred meaning which is selected by the operator. In addition, AFEI is able to express facial expressions according to its utterance. For example, when AFEI says "Be quiet!", it executes the facial expression “anger”. Also, AFEI is able to call students' name individually, because the names of the students who participate in the class are recorded in advance. Here, female voice that was recorded beforehand are used as AFEI's voice. The operator can execute these interactive behaviors easily by using a simple operation interface. In addition, AFEI apparently seems to conduct classes and interactions with students autonomously because AFEI is performed by the tele-operation.

\section{Operation Interface}

Fig. 7 shows an operation interface for an operator. As shown in the figure, there are many kinds of icons that correspond to robot's behaviors and utterances. It mainly consists of the following four parts; (a) the part for conducting a class, (b) the part for brief interaction, (c) the part for controlling the facial expressions, and (d) the part for controlling the head and eye movements. In part (a), there are icons to progress a class and to execute AFEI's utterances for explanations.

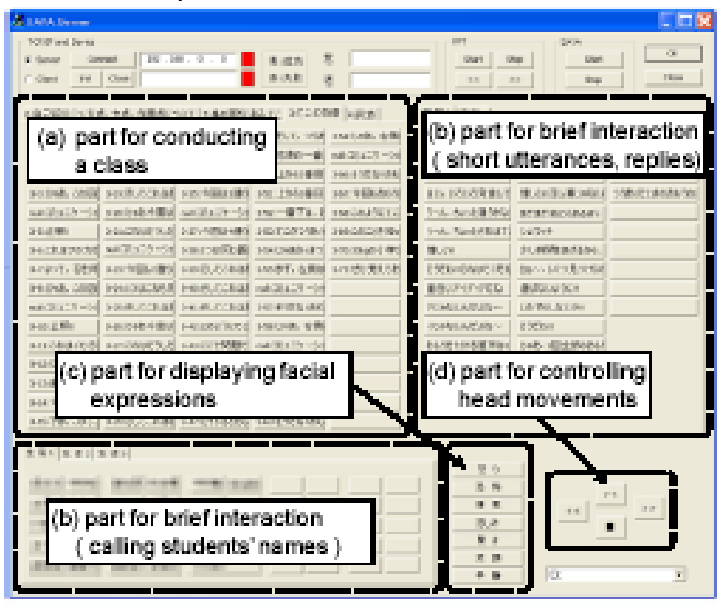

Figure 7. Operation interface.

The evaluation result by the training system analysis for 6 e-organization portals is shown as Fig. 8, in which A, B, C mean three cases.

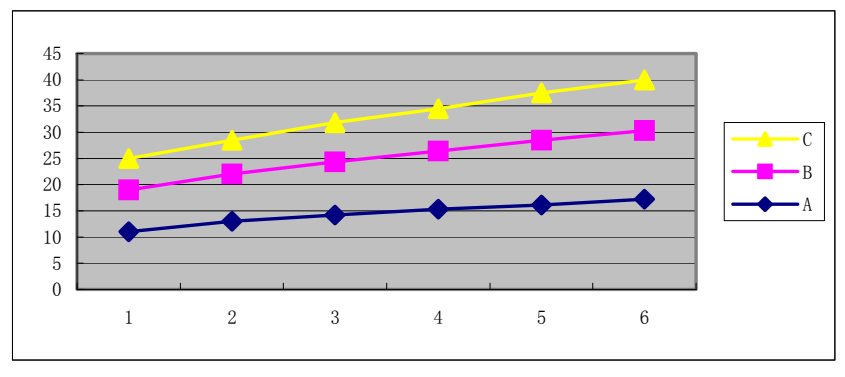

Figure 8. Evaluation result.

\section{REFERENCES}

[1] Anderson, P., What Is Web 2.0? Ideas, Technologies and Implications for Education, JISC TechWatch report, 2007.

[2] M. ISHII, “A robot teaching method using Hyper Card system” , Robot and Human Communication, pp.410 - 413, 1992.

[3] Mekid, S., Schlegel, T., Aspragathos, N., Teti, R. (2007), Foresight formulation in innovative production, automation and control systems, foresight, vol. 9, no. 5, pp. 35 - 47, 2007.

[4] Hvilshøj, M., Bøgh, S., Madsen, O., Kristiansen, M. (2010). Calibration Techniques for Industrial Mobile Manipulators: Theoretical configurations and Best practices, Proceedings of International Symposium on Robotics (ISR).

[5] Y. Nakamura, H. Hanafusa, "Inverse Kinematic Solutions with Singularity Robustness for Robot Manipulator Control” , Journal of Dynamic Systems, Measurement and Control, Vol.108, pp.163-71, 1986.

[6] Lyytinen, K. and Yoo, Y., .Issues and Challenges in Ubiquitous Computing., Communications of the ACM, Vol. 45, No. 12, 2002a. 